Research Paper

\title{
Colon Cancer Associated Transcript-1 (CCAT1) Expression in Adenocarcinoma of the Stomach
}

\author{
Ido Mizrahi ${ }^{*}$, Haggi Mazeh ${ }^{1 *}$, Ronit Grinbaum ${ }^{1}$, Nahum Beglaibter ${ }^{1}$, Michael Wilschanski², Vera Pavlov², \\ Muchamad Adileh ${ }^{3}$, Alexander Stojadinovic ${ }^{4}$, Itzhak Avital ${ }^{4}$, Ali Osmay Gure ${ }^{5}$, David Halle ${ }^{2}$, Aviram \\ Nissan $2,3 \bowtie$ \\ 1. Department of Surgery, Hadassah-Hebrew University Medical Center, Mount Scopus, Jerusalem, Israel; \\ 2. The Surgical Oncology Laboratory, Department of Surgery, Hadassah-Hebrew University Medical Center, Mount Scopus, Jerusalem, \\ Israel; \\ 3. Department of Surgery, Hadassah-Hebrew University Medical Center, Ein Kerem, Jerusalem, Israel; \\ 4. Bon Secours Cancer Institute, Richmond, VA, USA; \\ 5. Department of Molecular Biology and Genetics, Bilkent University, Ankara, Turkey. \\ * Both authors contributed equally to the preparation of this study.
}

$\square$ Corresponding author: Aviram Nissan, MD. Head, Department of Surgery, Hadassah Hebrew University Medical Center, Ein Kerem, POB 12000, Kyriat Hadassah, Jerusalem, Israel 91120. Telephone: +972-2-6779500 Fax: +972-2-6779510 e-mail: anissan@hadassah.org.il.

(C) Ivyspring International Publisher. This is an open-access article distributed under the terms of the Creative Commons License (http://creativecommons.org/ licenses/by-nc-nd/3.0/). Reproduction is permitted for personal, noncommercial use, provided that the article is in whole, unmodified, and properly cited.

Received: 2014.09.17; Accepted: 2014.10.15; Published: 2015.01.01

\begin{abstract}
Background: Long non-coding RNAs (IncRNAs) have been shown to have functional roles in cancer biology and are dys-regulated in many tumors. Colon Cancer Associated Transcript -1 (CCATI) is a IncRNA, previously shown to be significantly up-regulated in colon cancer. The aim of this study is to determine expression levels of CCAT1 in gastric carcinoma (GC).

Methods: Tissue samples were obtained from patients undergoing resection for gastric carcinoma $(n=19)$. For each patient, tumor tissue and normal appearing gastric mucosa were taken. Normal gastric tissues obtained from morbidly obese patients, undergoing laparoscopic sleeve gastrectomy served as normal controls $(n=19)$. A human gastric carcinoma cell line (AGS) served as positive control. RNA was extracted from all tissue samples and CCATI expression was analyzed using quantitative real time-PCR (qRT-PCR).

Results: Low expression of CCATI was identified in normal gastric mucosa samples obtained from morbidly obese patients [mean Relative Quantity $(R Q)=1.95 \pm 0.4$ ]. AGS human gastric carcinoma cell line showed an elevated level of CCAT1 expression $(R Q=8.02)$. Expression levels of $C C A T 1$ were approximately 10.8 fold higher in GC samples than in samples taken from the negative control group $(R Q=21.1 \pm 5$ vs. $R Q=1.95 \pm 0.4$, respectively, $p<0.001)$. Interestingly, CCAT1 expression was significantly overexpressed in adjacent normal tissues when compared to the negative control group $(R Q=15.25 \pm 2$ vs. $R Q=1.95 \pm 0.4$, respectively, $p<0.001)$. Tissues obtained from recurrent $\mathrm{GC}$ cases showed the highest expression levels $(R Q=88.8 \pm 31 ; p<0.001)$. Expression levels increased with tumor stage (T4- 36.4 $\pm 15, T 3-16.1 \pm 6, T 2-4.7 \pm 1$ ), however this did not reach statistical significance $(p=0.2)$. There was no difference in CCAT1 expression between intestinal and diffuse type $G C(R Q=22.4 \pm 7$ vs. $22.4 \pm 16$, respectively, $p=0.9)$. Within the normal gastric tissue samples, no significant difference in CCATl expression was observed in helicobacter pylori negative and positive patients $(R Q=2.4 \pm 0.9$ vs. $0.93 \pm 0.2$, respectively, $\mathrm{P}=0.13)$.

Conclusion: CCATI is up-regulated in gastric cancer, and may serve as a potential bio-marker for early detection and surveillance.
\end{abstract}

Key words: Gastric cancer, CCAT1, gastrectomy, biomarker, long non-coding RNA. 


\section{Introduction}

Worldwide, gastric cancer is the fourth most common cancer and the second leading cause of cancer death. While the incidence rates have been decreasing in the United States, they have been steadily increasing in East Asia and South America [1, 2]. Major risk factors associated with the development of gastric carcinoma include the presence of Helicobacter Pylori infection, high salt diet, rare inherited disorders, and male gender [3].

Several genetic alterations have been identified as carcinogenic changes involved in the development of gastric carcinoma. These changes can be roughly divided into the activation of proto-oncogenes (c-met, k-sam, c-erbB2), the inactivation of tumor suppressor genes (p16, p53), reduction or loss in the cell adhesion molecule E-cadherin, telomerase reactivation, and microsatellite instability [4]. Nonetheless, exact mechanisms at the molecular level remain largely unknown.

Studies focusing on finding specific molecular biomarkers for the detection of different cancers, have found recently long non-coding RNA molecules (lncRNA) to have functional roles in cancer biology. Possible mechanisms by which these lncRNAs influence carcinogensis, may be by binding to, and altering the function of proteins known to be involved in tumor biology such as $p 53$, or by binding to promoter areas of different genes and dys-regulating their expression [5-7]. Colon Cancer Associated Transcript -1 (CCAT1) is a lncRNA, up-regulated across the adenoma-carcinoma sequence in colon cancer and to a lesser degree, up-regulated in other tumor types [8,9]. CCAT1 is a 2628 nucleotide lncRNA located on chromosome 8q24.21 in an intergenic area described before as a "hot spot" harboring multiple genetic alternations in both colon and prostate cancer. It is located in the vicinity of c-MYC, a well-known transcription factor [10, 11]. It was discovered using Representational Difference Analysis (RDA), cDNA cloning, and rapid amplification of cDNA ends (RACE) [9].

Recently, CCAT1 has been found to be highly expressed in gastric cancer tissues when compared with adjacent, normally appearing tissues. However, no comparison was made to normal gastric tissues obtained from healthy controls [12]. These data, if replicated by others supports a potential role for CCAT1 as a biomarker for gastric cancer. The aims of this study were:

- To validate our preliminary (unpublished data) and the data, obtained by others, showing upregulation of CCAT1 in gastric cancer.

- To characterize CCAT1 expression levels through various tumor stages.
- To compare CCAT1 expression to a cohort of patients without malignant or premalignant changes.

\section{Methods}

\section{Patients}

This is a pathological and molecular study designed and conducted on fresh-frozen human tissues. The study was approved by the Institutional Independent Ethical Committee (IBE, Helsinki Committee; Protocol HMO-0253-10). Patients eligible for the study were offered participation and signed a written informed consent. Eligible patients were patients with histological diagnosis of gastric adenocarcinoma scheduled to undergo gastric resection with curative intent. Patients with evidence of metastatic disease were excluded and directed towards palliative medical therapy. To be eligible for the control group, patients had to meet the national criteria for surgical treatment of morbid obesity and to be scheduled for laparoscopic sleeve gastrectomy.

Other eligibility criteria: included: Patients above the age of 18 years capable of providing informed consent. Patients with gastric cancer who were not treated by chemotherapy, nor by radiation therapy before surgery. Patients with recurrent tumor presenting at least 24 months after completion of previous therapy were allowed.

Patients with invasive cancers other than adenocarcinoma of the stomach were excluded.

Human gastric cancer cell line (AGS, Cell line human, 89090402, Sigma-Aldrich) served as a positive control.

\section{Tissue procurement}

Immediately following surgical resection, the resected specimen was delivered fresh to the Department of Pathology, where, under the supervision of an independent pathologist, a small portion of resected tissue was snap frozen in liquid nitrogen for future RNA extraction. Tissues were obtained from the primary lesion, and from normal appearing mucosa adjacent to the primary tumor site, two biopsies each. For the negative control group, two biopsies were obtained from random areas of the resected stomach. Final pathology reports were reviewed for all patients to determine either tumor stage or, for the negative control group, Helicobacter pylori, and gastritis status.

\section{Total RNA isolation from tissues}

Total RNA was extracted using the miRvana ${ }^{\circledR}$ isolation kit (Ambion Inc., Austin, TX) in accordance with manufacturer instructions. Weighed tissues were thoroughly crushed on dry ice and disrupted with 1 
$\mathrm{ml} / 50-100 \mathrm{mg}$ tissue, denaturizing lysis buffer using a polytron tissue homogenizer. RNA concentration was measured with NanoDrop ${ }^{\circledR}$ Spectrophotometer (ND-100, NanoDrop Technologies, Wilmington, DE) and stored at $-80^{\circ} \mathrm{C}$ until further use.

\section{Synthesis of cDNA}

Following DNase treatment, cDNA synthesis was performed using random primer (Roche Diagnostics $\mathrm{GmbH}$, Mannheim, Germany) added to $10 \mu \mathrm{l}$ of RNA. After incubation, $1 \mu \mathrm{l}$ of reverse transcriptase (SuperScript II Reverse Transcriptase 200 U/ $\mu$ l, Invitrogen, Carlsbad, CA) was added. The cDNA was stored at $-20^{\circ} \mathrm{C}$ until used for qRT-PCR.

\section{Real time quantitative PCR}

Primers used were: CCAT1 (custom designed by Applied Biosystems Inc., Foster City, CA): CCAT1-Forward

TCACTGACAACATCGACTTTGAAG CCAT1-Reverse

GGAGAAAACGCTTAGCCATACAG

GAPDH was used as a control gene.

CCAT1 RNA was normalized to GAPDH-RNA content using ABI 7500 SDS software, v1.2.2 (Applied Biosystems Inc., Foster City, CA). Positive and negative controls, as well as samples with no DNA were included in every qRT-PC experiment. PCR reactions were performed using ABI qRT-PCR thermocycler (7500 Real Time PCR System, Applied Biosystems Inc., Foster City, CA). The qRT-PCR program was run for 40 cycles, following an initial incubation at $95^{\circ} \mathrm{C}$, $10 \mathrm{~min}$. Each cycle consisted of $95^{\circ} \mathrm{C} \times 15 \mathrm{sec}$. and $60^{\circ} \mathrm{C} \times 1 \mathrm{~min}$. Results were analyzed as relative quantity (RQ) expression of CCAT1.

\section{Statistical analysis}

Summary statistics were obtained using established methods. Associations between categorical factors were studied with Fisher's exact test or Chi-squared test, as appropriate. Continuous variables between study groups were compared using the T-test (two-sided). Statistical analysis was performed using IBM-SPSS ${ }^{\circ}$ statistical package Version 20.0 (SPSS Inc. Chicago, IL). A p value $<0.05$ was considered significant.

\section{Results}

Tissues were obtained, as detailed above, from patients that underwent surgery for gastric cancer $(n=19)$, and from patients in the negative control group $(n=19)$, that underwent laparoscopic sleeve gastrectomy for treatment of morbid obesity. Overall, RNA extraction and cDNA production was successful in $100 \%(19 / 19)$ of patients with GC, and in $94 \%$
$(18 / 19)$ of patients in the negative control group.

The patients in the study group had a mean age of $64.5 \pm 3$ years, and consisted of 14 males $(73 \%)$, and 5 females $(27 \%)$. Twelve patients $(63 \%)$ had intestinal type GC and seven patients (37\%) had diffuse type GC (Table 1). Expression levels of CCAT1 were approximately 10.8 fold higher in GC samples than in samples taken from the negative control group $(\mathrm{RQ}=21.1 \pm 5$ vs. $\mathrm{RQ}=1.95 \pm 0.4$, respectively, $\mathrm{p}<0.001$, Table 1). As we have observed before in colon cancer, CCAT1 was significantly up-regulated in normal appearing tissues adjacent to the tumor site when compared to normal controls $(\mathrm{RQ}=15.25 \pm 2$ vs. $R Q=1.95 \pm 0.4$, respectively, $p<0.001$ ). Interestingly enough, CCAT1 expression levels in histologically normal appearing tissues adjacent to the tumor site exceeded its expression levels in the tumor itself (Figure 1). Samples taken from patients with recurrent GC (sample \#343, sample \#443) showed the highest levels of CCAT1 expression $(R Q=88.8 \pm 31)$. There was a correlation between CCAT1 expression and tumor penetration into the gastric wall as expressed by T-stage (T4- RQ=36.4 $\pm 15, \quad T 3-R Q=16.1 \pm 6, \quad T 2-$ $\mathrm{RQ}=4.7 \pm 1$ ), however, this correlation did not reach statistical significance $(p=0.2$, Figure 2$)$. There was no difference in CCAT1 expression levels between intestinal and diffuse type gastric cancer $(\mathrm{RQ}=22.4 \pm 7$ vs. $22.4 \pm 16$, respectively, $p=0.9$ ). No difference was found in CCAT1 expression levels between proximal gastro-esophageal junction tumors, and distal gastric tumors ( $41 \pm 22$ vs. $18 \pm 6$, respectively, $\mathrm{p}=0.23$ ).

Table 1: Clinical data, and CCAT1 expression levels in tumor \& adjacent normal tissue.

\begin{tabular}{|c|c|c|c|c|c|c|c|}
\hline $\mathrm{N}$ & $\begin{array}{l}\text { Sample } \\
\#\end{array}$ & Gender & Age & $\begin{array}{l}\text { Tumor } \\
\text { AJCC } \\
\text { stage }\end{array}$ & $\begin{array}{l}\text { His- } \\
\text { to-pathologic } \\
\text { al subtype }\end{array}$ & $\begin{array}{c}\text { RQ } \\
\text { CCAT1 In } \\
\text { Tumor } \\
\end{array}$ & $\begin{array}{c}\text { RQ CCAT1 } \\
\text { In adjacent } \\
\text { normal }\end{array}$ \\
\hline 1 & 343 & M & 77 & $\begin{array}{l}\text { T4N1M0 } \\
\text { Recurrence }\end{array}$ & Intestinal & 57.56 & $\mathrm{~N} / \mathrm{A}$ \\
\hline 2 & 443 & M & 46 & $\begin{array}{l}\text { T4N1M0 } \\
\text { Recurrence }\end{array}$ & Diffuse & 120.21 & 25.28 \\
\hline 3 & 496 & M & 75 & T3N1M1 & Intestinal & 2.91 & 5.53 \\
\hline 4 & 540 & $\mathrm{M}$ & 65 & T4N3M0 & Diffuse & 5.63 & 42.11 \\
\hline 5 & 560 & $\mathrm{~F}$ & 48 & T4N3M0 & Diffuse & 1.65 & 12.73 \\
\hline 6 & 939 & M & 58 & T3N2M0 & Intestinal & 8.97 & 42.47 \\
\hline 7 & 1029 & M & 65 & T3N0M0 & Intestinal & 34.51 & 14.11 \\
\hline 8 & 1064 & $\mathrm{M}$ & 60 & $\mathrm{~T} 2 \mathrm{~N} 2 \mathrm{M} 0$ & Intestinal & 10.61 & 39.67 \\
\hline 9 & 621 & $\mathrm{M}$ & 73 & T4N0M0 & Diffuse & 2.7 & 15.7 \\
\hline 10 & 743 & $\mathrm{M}$ & 82 & T4N2M0 & Diffuse & 23.47 & 23.18 \\
\hline 11 & 826 & $\mathrm{M}$ & 75 & T2N0M0 & Intestinal & 7.17 & 6.52 \\
\hline 12 & 865 & $\mathrm{~F}$ & 34 & T2N1M0 & Intestinal & 5.53 & 0.09 \\
\hline 13 & 882 & $\mathrm{M}$ & 36 & T4N3M0 & Diffuse & 2.08 & 4.49 \\
\hline 14 & 919 & $\mathrm{~F}$ & 72 & T3N1M0 & Diffuse & 4.89 & 8.51 \\
\hline 15 & 978 & $\mathrm{~F}$ & 86 & T3N2M0 & Intestinal & 13.78 & 0.89 \\
\hline 16 & 982 & $\mathrm{M}$ & 80 & T3N2M0 & Intestinal & 4.18 & 6.95 \\
\hline 17 & 1026 & $\mathrm{~F}$ & 81 & T3N1M0 & Intestinal & 43.62 & 29.48 \\
\hline 18 & 1063 & $\mathrm{M}$ & 49 & T4N1M0 & Intestinal & 78.01 & 0.63 \\
\hline 19 & 1184 & $\mathrm{M}$ & 78 & T2N1M0 & Intestinal & 1.92 & 9.7 \\
\hline
\end{tabular}


Patients in the negative control group $(n=19)$ had an average age of $43.3 \pm 2$ years, and consisted of 9 men (50\%) and 9 women (50\%). Low CCAT1 expression levels were detected in this group ( $R Q=1.95 \pm 0.4)$. Twelve patients $(66 \%)$ had histological evidence of gastritis, and six patients (34\%) were positive for the presence of Helicobacter Pylori. No significant difference in CCAT1 expression was observed in helicobacter pylori negative, and positive patients $(\mathrm{RQ}=$ $2.4 \pm 0.9$ vs. $0.93 \pm 0.2$, respectively, $p=0.13$ ), or in gastritis negative and positive patients $(R Q=2.42 \pm 0.9$ vs. $0.9 \pm 0.2, p=0.28$ ). Table 2 summarizes clinical data, and CCAT1 expression levels in the negative control group. Figure 3 summarizes CCAT1 expression levels in the different study groups.

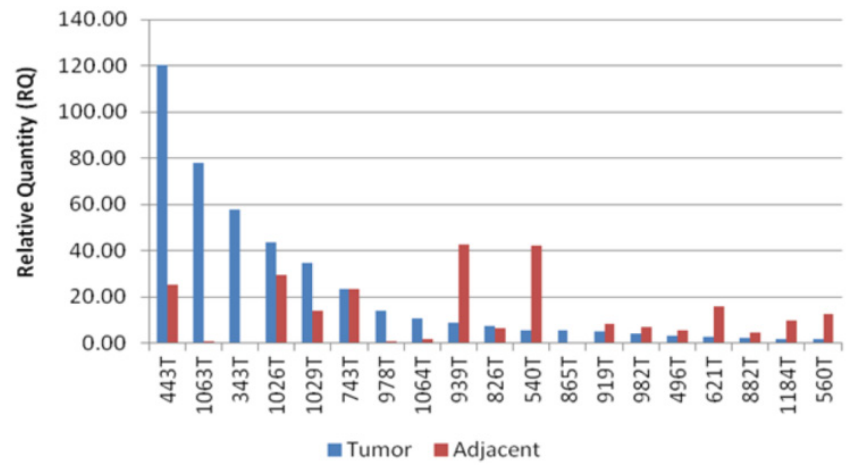

Figure 1: CCAT1 expression levels in GC tumor samples and in adjacent normal samples.

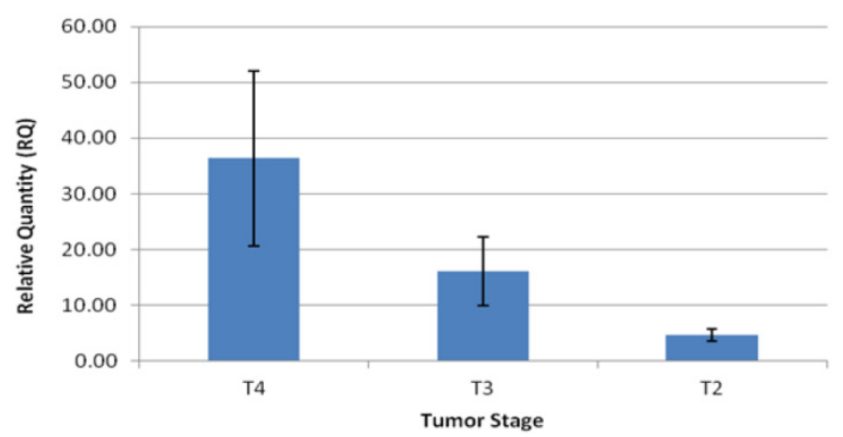

Figure 2: CCATl expression levels according to tumor (AJCC-T) stage.

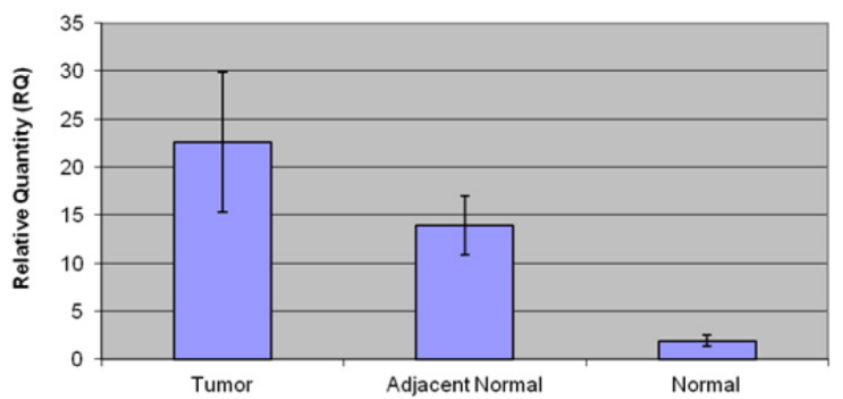

Figure 3: CCAT1 expression levels in the different study groups.
Table 2: Clinical data and CCAT1 expression levels in the negative control group.

\begin{tabular}{lllllll}
\hline N & $\begin{array}{l}\text { Sample } \\
\#\end{array}$ & Gender & Age & Gastritis & Helicobacter pylori & RQ \\
CCAT1
\end{tabular}

\section{Discussion}

Gastric cancer prognosis is in close correlation with tumor stage at diagnosis.

Primarily due to early detection of the disease, and with the aid of nationwide screening programs, postoperative survival rates have increased in Japan and Korea. In the Western world, however, more than $80 \%$ of patients at diagnosis have an advanced gastric cancer with poor prognosis $[13,14]$. Improvement of survival rates is dependent upon, earlier diagnosis, closer monitoring of populations at risk, and new therapeutic strategies. Hence, the obvious need in improving diagnostic sensitivity, among which with bio-molecular tests.

Over the last few decades, research has focused on the role of protein-coding genes in the pathogenesis of diseases [15]. However, in recent years, evidence from whole genome and transcriptome sequencing suggests that long noncoding RNAs (lncRNAs) which belong to the noncoding portions of the genome, play a key role in oncogenesis [16]. Accumulating reports of dys-regulated lncRNA expression in numerous cancer types imply that lncRNAs may act as potential onco- or tumor-suppressor RNAs [17, 18]. Thus, the attention of research is now shifting to one of the most common but least well-understood RNA species: IncRNAs [19].

Most of the lncRNAs studied to date in various cancers are overexpressed, which indicates an oncogene-like role of lncRNAs in cancer biology [20]. Such a lncRNA is CCAT1, previously found to be significantly overexpressed in colon cancer. In these studies, 
CCAT1 expression in colon cancer was approximately 235 fold higher than in normal colonic tissue. Up-regulation was evident in pre-malignant conditions and through all disease stages, including advanced metastatic disease suggesting a role in both tumorigenesis and the metastatic process [8, 9]. CCAT1 was also up-regulated in cell lines derived from non-small cell lung cancer and pancreatic cancer. Nevertheless, exact molecular mechanisms in which CCAT1 is involved in carcinogenesis are yet to be found. A recent study by Feng et al., studying CCAT1 expression levels in gastric cancer, found that c-MYC directly binds to the E-box element in the promoter region of CCAT1, and when ectopically expressed, increases promoter activity and expression of CCAT1 in gastric cancer tissues [12]. In addition, nucleotide substitutions in the E-box element abrogated c-MYC-dependent promoter activation. Moreover, abnormally expressed CCAT1 promoted cell proliferation and migration. However, Feng et al. compared GC tissues to tumor adjacent, appearing normal tissues, and not to normal healthy controls. Our study aimed to further characterize CCAT1 expression in GC, and to compare expression levels to normal healthy controls.

Our results show expression levels of CCAT1 approximately 10.8 fold higher in GC samples than in samples taken from healthy individuals in the negative control group $(R Q=21.1 \pm 5$ vs. $R Q=1.95 \pm 0.4$, respectively, $\mathrm{p}<0.001)$. These results further support previous results by Feng et al., showing an approximate 4 fold higher expression level of CCAT1 in GC samples when compared to adjacent normal tissues. As we have seen before in colon cancer tissues, CCAT1 expression might be higher in the histologically normal appearing tissues around the tumor than within the tumor tissue itself. This observation was previously confirmed by in-situ hybridization in order to rule out contamination by shedding of cancer cells.

In our study we compared tissue samples taken from GC tissues not only to histologically normal-appearing tissues adjacent to the tumor site, but also to normal gastric tissues taken from patients with no evidence of GC undergoing sleeve gatsrectomy due to morbid obesity. Interestingly, CCAT1 expression was significantly overexpressed in tumor-adjacent normal tissues when compared to healthy controls $(R Q=15.25 \pm 2$ vs. $R Q=1.95 \pm 0.4$, respectively, $\mathrm{p}<0.001$ ). This might be a cause of underlying pre-cancerous molecular events occurring in adjacent normal tissue causing a potential "field effect". Another explanation might be the diffuse nature in which some GC spread. However in a subgroup analysis, no difference in expression levels was found between the different histo-pathological, intestinal and diffuse subtypes $(R Q=22.4 \pm 7$ vs. $22.4 \pm 16$, respectively, $\mathrm{p}=0.9$ ).

As expected, the majority of the patients in our study $(79 \%)$ were diagnosed with GC at advanced tumor stage. Of note, patients treated with neo-adjuvant chemotherapy were excluded in order to eliminate potential chemotherapy effect on CCAT1 expression. CCAT1 expression level showed a trend towards correlation with advancement in tumor (AJCC-T) stage $(\mathrm{T} 4-\mathrm{RQ}=36.4 \pm 15, \mathrm{~T} 3-\mathrm{RQ}=16.1 \pm 6$, T2$\mathrm{RQ}=4.7 \pm 1, \mathrm{p}=0.2)$. We also demonstrated that samples taken from patients with recurrent GC, showed the highest expression of CCAT1. Similar results were presented by Feng et al [12]. Despite this correlation between mean CCAT1 expression levels and tumor stage, it is important to note that ten patients in the GC group had a higher expression levels in adjacent normal tissue than in tissues taken from the tumor itself (table 1, figure 1). In addition to technical errors that might explain these results, there are several other potential explanations. As stated above, this observation might be a result of potential "field effect" in adjacent normal tissues or a consequence of the diffuse nature in which some GC spread. Another theoretical explanation is that CCAT1 expression may fluctuate through different stages of tumorigenesis; reaching its peak in early stages and subsequently silenced at later stages. We were not able to enroll patients with pre-cancerous conditions or early stage GC into our study, therefore no statement could be made regarding expression levels in early GC stages. We believe that CCAT1 levels indeed increase with the advancement in tumor stage and that a larger cohort would have been able to achieve statistical significance.

The presence of Helicobacter pylori infection is considered a major risk factor for the development of GC [21]. The association between HP and CCAT1 was not previously studied. We hypothesized that CCAT1 expression would be higher in normal gastric tissues obtained from patients, in the control group, infected by Helicobacter pylori due to the inflammatory process. Our results showed that in fact, there is no significant difference in CCAT1 expression in Helicobacter pylori negative, and positive patients $(R Q=2.4 \pm 0.9$ vs. $0.93 \pm 0.2$, respectively, $\mathrm{p}=0.13$ ). Hypothetically, pre-cancerous processes related to Helicobacter pylori infection might have still not occurred in these patients.

This study has several limitations. The study group consisted of a relatively small number of patients, most of which were in advanced stages of the disease. Perhaps with a larger and more diverse cohort, we would have been able to show a significant 
increase in CCAT1 expression with tumor advancement. Significant gender and age differences between the two study groups are of potential bias. Theoretically, CCAT1 up-regulation might be influenced by age and male gender. Including patients of older age and more patients of male gender in the control group would probably be more methodologically appropriate. Another limitation might be the absence of lymph nodes or metastasis as part of the tissues analyzed. Comparison of CCAT1 expression in lymph nodes and histo-pathological findings should be the subject for future research.

Despite its limitations, our study is the second study to date reporting high levels of CCAT1 expression in gastric cancer, and the only study comparing expression levels between GC tissues and normal, healthy gastric tissues taken from subjects without cancer. We believe that CCAT1 has the potential of serving as a biomarker for GC, improving diagnosis and surveillance, as well as a target for innovative molecular therapies. Future large scale studies are needed to examine CCAT1 expression in all stages of GC, and to study the exact molecular mechanism in which CCAT1 is involved in GC oncogenesis.

\section{Competing Interests}

The authors have declared that no competing interest exists.

\section{References}

1. Jemal A, Siegel R, Xu J, Ward E. Cancer statistics, 2010. CA Cancer J Clin. 2010;60:277-300.

2. Moore MA, Attasara P, Khuhaprema T, Le TN, Nguyen TH, Raingsey PP, et al. Cancer epidemiology in mainland South-East Asia - past, present and future. Asian Pacific journal of cancer prevention : APJCP. 2010;11 Suppl 2:67-80.

3. de Martel C, Forman D, Plummer M. Gastric cancer: epidemiology and risk factors. Gastroenterol Clin North Am. 2013;42:219-40.

4. Nobili S, Bruno L, Landini I, Napoli C, Bechi P, Tonelli F, et al. Genomic and genetic alterations influence the progression of gastric cancer. World J Gastroenterol. 2011;17:290-9.

5. Spizzo R, Almeida MI, Colombatti A, Calin GA. Long non-coding RNAs and cancer: a new frontier of translational research? Oncogene. 2012;31:4577-87.

6. Hung T, Wang Y, Lin MF, Koegel AK, Kotake Y, Grant GD, et al. Extensive and coordinated transcription of noncoding RNAs within cell-cycle promoters. Nat Genet. 2011;43:621-9.

7. Moran VA, Perera RJ, Khalil AM. Emerging functional and mechanistic paradigms of mammalian long non-coding RNAs. Nucleic acids research. 2012;40:6391-400.

8. Alaiyan B, Ilyayev N, Stojadinovic A, Izadjoo M, Roistacher M, Pavlov V, et al. Differential expression of colon cancer associated transcript1 (CCAT1) along the colonic adenoma-carcinoma sequence. BMC Cancer. 2013;13:196.

9. Nissan A, Stojadinovic A, Mitrani-Rosenbaum S, Halle D, Grinbaum R, Roistacher $M$, et al. Colon cancer associated transcript-1: A novel RNA expressed in malignant and pre-malignant human tissues. Int J Cancer. 2011.

10. Zanke BW, Greenwood CM, Rangrej J, Kustra R, Tenesa A, Farrington SM, et al. Genome-wide association scan identifies a colorectal cancer susceptibility locus on chromosome 8q24. Nat Genet. 2007;39:989-94.

11. Yeager $\mathrm{M}$, Orr $\mathrm{N}$, Hayes RB, Jacobs $\mathrm{KB}$, Kraft $\mathrm{P}$, Wacholder $\mathrm{S}$, et al. Genome-wide association study of prostate cancer identifies a second risk locus at 8q24. Nat Genet. 2007;39:645-9.

12. Yang $\mathrm{F}$, Xue X, Bi J, Zheng L, Zhi K, Gu Y, et al. Long noncoding RNA CCAT1, which could be activated by c-Myc, promotes the progression of gastric carcinoma. Journal of cancer research and clinical oncology. 2013;139:437-45.

13. Roukos DH. Current status and future perspectives in gastric cancer management. Cancer Treat Rev. 2000;26:243-55.

14. Herbreteau E, Jooste V, Hamza S, Lepage C, Faivre J, Bouvier AM. Trends in the management of gastric cancer over a 32-year period: a French population-based study. Gastric cancer : official journal of the International Gastric Cancer Association and the Japanese Gastric Cancer Association. 2014.

15. Tsai MC, Spitale RC, Chang HY. Long intergenic noncoding RNAs: new links in cancer progression. Cancer Res. 2011;71:3-7.

16. Mattick JS. RNA regulation: a new genetics? Nat Rev Genet. 2004;5:316-23.

17. Gibb EA, Brown CJ, Lam WL. The functional role of long non-coding RNA in human carcinomas. Molecular cancer. 2011;10:38.

18. Huarte M, Rinn JL. Large non-coding RNAs: missing links in cancer? Hum Mol Genet. 2010;19:R152-61.

19. Derrien T, Johnson R, Bussotti G, Tanzer A, Djebali S, Tilgner H, et al. The GENCODE v7 catalog of human long noncoding RNAs: analysis of their gene structure, evolution, and expression. Genome Res. 2012;22:1775-89.

20. Qiu MT, Hu JW, Yin R, Xu L. Long noncoding RNA: an emerging paradigm of cancer research. Tumour biology : the journal of the International Society for Oncodevelopmental Biology and Medicine. 2013;34:613-20.

21. Lian G, Wei C, Wang D, Cui M, Wang Z, Liu X, et al. Protein profiling of Helicobacter pylori-associated gastric cancer. Am J Pathol. 2014;184:1343-54. 